\title{
OEIS COMPLEX: A RARE CASE REPORT
}

Bharadwaj Mishra1, Lakshmi Sujani C.H²

\section{HOW TO CITE THIS ARTICLE:}

Bharadwaj Mishra, Lakshmi Sujani CH. "OEIS complex: a rare case report". Journal of Evolution of Medical and Dental Sciences 2013; Vol2, Issue 48, December 02; Page: 9410-9414.

ABSTRACT: The OEIS complex comprises of a combination of defects including Omphalocele, Exstrophy of the cloaca, Imperforate anus and Spinal defects. It represents the most severe manifestation of EES (exstrophy - epispadias sequence). It results from defective blastogenesis in 4th week of gestation, resulting in improper closure of anterior abdominal wall and defective development of cloaca and urogenital septum. A third gravida with previous normal obstetric history was diagnosed by prenatal ultrasound to have a grossly malformed fetus. It was provisionally diagnosed as OEIS complex by non- visualisation of bladder, presence of omplalocele, limb and spinal defects in the ultrasound.Hysterotomy confirmed the defects in the dead baby, which was further confirmed by autopsy, fetal CT scan and X-ray. Prognosis depends on severity of bladder exstrophy and neural tube defects. Likewise, management varies from interruption of pregnancy in severe cases to appropriate perinatal management in less severe cases by team of obstetricians, neonatologists, pediatric surgeons and neurosurgeons.

KEYWORDS: Exstrophy of cloaca, Omphalocele, Imperforate anus, Epispadias

INTRODUCTION: OEIS (Omphalocele Exstrophy of cloaca Imperforate anus and Spinal defects) complex is a rare fetal polymalformation of uncertain etiology, has been regarded as sporadic in nature with a low recurrence risk.It results from abnormal closure of ventricular abdominal due to failure of convergence of cephalo-caudal and lateral folidings of embryo during blastogenesis associated with defective development of cloaca and urogenital septum. OEIS complex represents most severe form of Exstrophy Epispadias Complex (EEC). EEC includes spectrum of urogenital abnormalities ranging from less severe Classical Bladder Exstrophy to most severe Exstrophy of cloaca. (1) It is a rare disorder affecting about 1 in 200, 000 to 400, 000 pregnancies. In the present case all the four classical features are seen in addition to gastrointestinal malformations and absence of gonads and internal genital organs.

CASE REPORT: An unbooked case of 29 year old third gravida (G3P2L2) came to OPD of OBGY dept of ASRAM medical college at 16 weeks of gestation for routine antenatal care. She was advised TIFA scan which revealed severely malformed fetus. Her obstetric history was significant for two previous $\mathrm{C}$-sections for recurring cause (CPD) having healthy children without any congenital anomalies. She had not taken folic acid and iron supplementation, no history of any other medications. There was no history of diabetes or any other medical disorders. No history of consanguity and no family history of congenital malformations. Not habituated to smoking nor addicted to alcohol.

On examination of the abdomen fundal height corresponding to 18 weeks was noticed. On vaginal examination cervix was posterior, firm, with os closed. Hematological and biochemical parameters were normal. She was advised TIFA scan. Ultrasound report showed a grossly malformed fetus of estimated gestational age 15 wks 4 days based on biometry (BPD, HC, FL). Scan also revealed an ill defined anterior abdominal wall defect through which abdominal contents 
herniated into extra embryonic coelom. Protruded organs formed a complex of large loculated fluid filled cystic spaces outside abdomen. Ultrasound also showed scoliosis deformity with short spine, meningocele at lumbosacral area, bilaterally dilated ventricles in head. Heart was intrathoracic with cardiac pulsations at $146 \mathrm{bpm}$. Both kidneys were visualized and were outside the abdominal wall in the herniated sac. Bladder was not visualized. Possibility of clubfoot deformity was there. Placenta was posterior with cord insertion not properly visualized. TORCH profile of the mother was normal. A provisional diagnosis of OEIS was made based on ultrasound description. She was admitted in the department of OBGY for termination of her pregnancy after explaining poor prognosis of fetus (IP REGD N0.2013040060) on 03-10-2013 Induction of abortion was not done due to prior two Csections. Patient was taken up for hysterotomy. Bilateral tubectomy was also done as she had two healthy children and was not intending to conceive again. Fetus presented as vertex and delivered as such, weighing 860gms.It was gasping and died immediately. Placenta was found adherent to uterine wall at previous scar line and hence removed manually. Fetus with placenta sent for autopsy (b.no.7589/13).

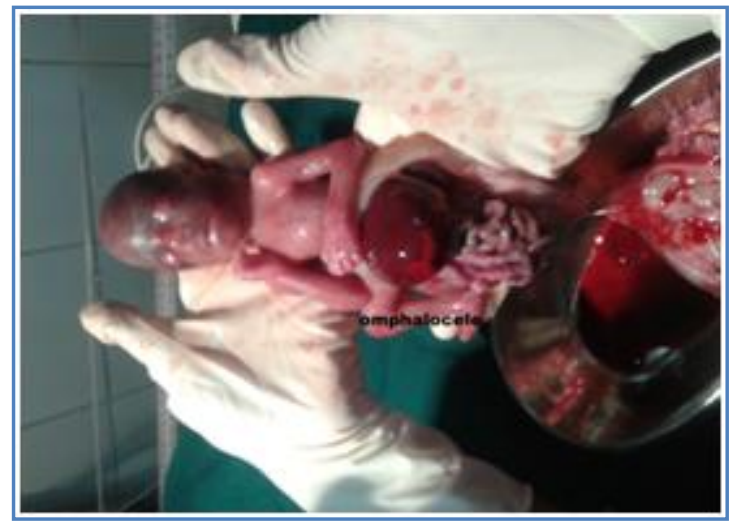

Fig.1: Post op picture showing omphalocele

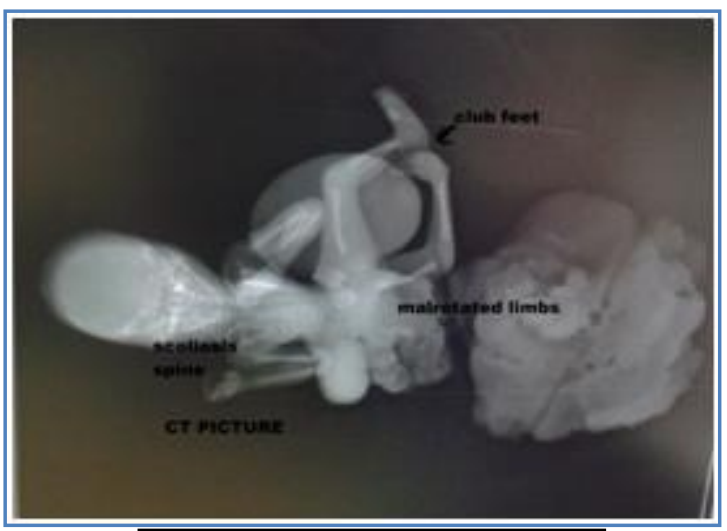

Fig. 3: CT picture of fetus

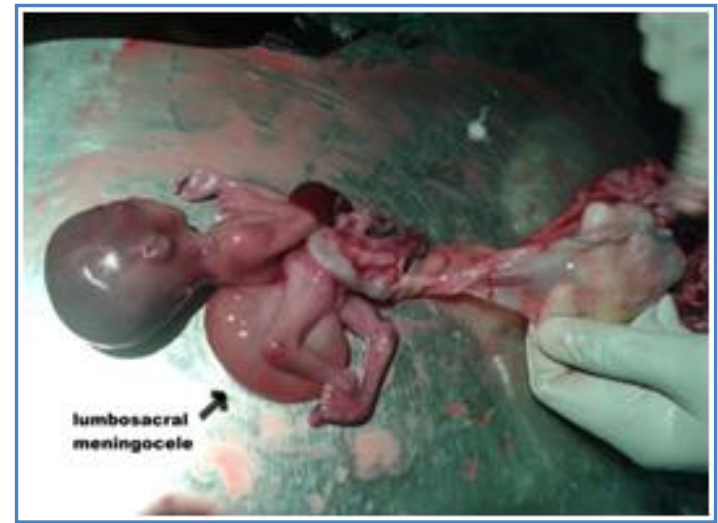

Fig.2: postop picture showing lumbosacral meningocele

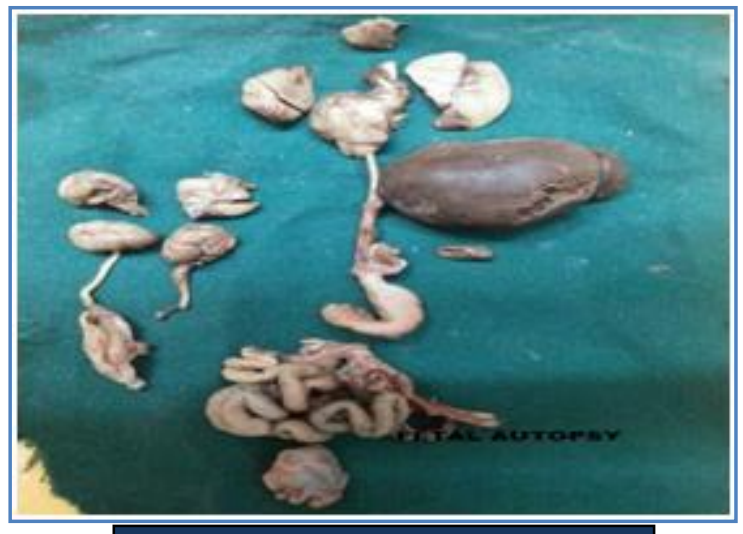

Fig.4: viscera in fetal autopsy 
Autopsy report on external examination showed a large head, a $12 \times 10 \mathrm{~cm}$ defect in anterior abdominal wall, exstrophy of bladder. Anal opening was absent in perineal region. External genitalia were absent. Lumbosacral meningocele was seen. Fetal spine showed scoliosis and both the limbs were rotated to one side. Umbilical cord was short (12 centimeters), showing two arteries and one vein. On internal examination, thoracic cavity was normal. There was malrotation of gastrointestinal system. Liver with coils of small intestine was seen on left side. Stomach, cecum with appendix and colon were on right side. Liver was enlarged. Gall bladder was not visualized. Intestines were dilated and anal canal was not seen. Both kidneys and adrenals were in their anatomical position. Ureters were identified. Exstrophy of bladder was seen. Size of head was large compared to its age. Brain was hypo plastic with ill formed gyri and sulci.

DISCUSSION: The first description of case was published by Littre et al in 1709 (2). The term OEIS complex was first used by Carey et al in 1978. He described a congenital syndrome with multiple malformations including omphalocele, exstrophy of bladder, imperforate anus and spinal defects (3). Previous terminologies used were Ectopia of cloaca, Vesico- intestinal fissure, Exstrophy splanchna. Subsequently in addition to the four classical manifestations, its association with genital renal and skeletal abnormalities like spina bifida, symphysis pubis diastasis and limb abnormalities were statistically demonstrated. (4)

The occurrence of exstrophy of the bladder appears to be more common (1:30 000 to 1:40 000) than exstrophy of cloaca (1:200.000 to 250.000) or pregnancies with OEIS complex (1:200.000 to $1: 400.000$ ) (5). The incidence of OEIS is probably higher because many cases are diagnosed incorrectly as omphalocele, which is the most prominent component of this malformation complex (6). A male predominance was observed in the original report by Carey et al. (7) Schinzel et al (1979) estimated that $10 \%$ of cloacal exstrophy occur in same sex twins and suggested that multiple pregnancies and early malformations like exstrophy are related (3). Karyotyping is usually normal according to Witters et al (8).

Etiology is unknown but several associations have been suggested. Most frequently it is of sporadic in occurrence. Possibility of teratogenic exposure with diazepam (9), diphenylhydantoin (3), genetic factors such as trisomy 18 (7), single gene defects in blastogenesis and mutations in homeobox genes such as HLXB9 (3) have been suggested. Higher incidence in monozygotic twins suggest a possible genetic contribution to occurrence of this multisystem defect (6). Recurrence of omphalocele or bladder exstrophy in siblings have been documented with a risk less than 1\% (4).

OEIS is considered to be a defect in blastogenesis, beginning in the first four weeks of human development. According to different authors, OEIS has probably a heterogeneous etiology and may result from a single localized defect in early caudal mesoderm at approximately 29 days of development and it is thought to lead to one of three defects (1):.failure of cloacal septation resulting in a common cloaca, breakdown of cloacal membrane resulting in omphalocele and exstrophy or incomplete vertebral fusion resulting in open neural defects.

Early diagnosis is possible by TIFA scan, which may show absence of filled up well circumscribed bladder, persistence of physiological herniation of intestinal contents beyond 8 to 10 weeks of pregnancy, omphalocele, associated spinal defects and minor criteria like lower extremities malformations, renal anomalies, ascites, widened pubic arch, narrow thorax, hydrocephalous, single umbilical artery. According to literature sonographic findings of OEIS complex have been 
documented but only few cases of prenatal diagnosis have been reported. In our case based of ultrasound, prenatal diagnosis is made and patient is informed of poor prognosis and counseled for termination of pregnancy.

Differential diagnosis includes omphalocele and gastroschisis. Both conditions are excluded from OEIS complex by presence of normally filling bladder in ultrasound in those conditions. There is significant overlap between OEIS complex and limb body wall complex (11) but OEIS complex is recognized by absent bladder. Others include Pentology of Cantrell which can be differentiated by its characteristic anterior thoracic defects and absence of spinal defects. Amniotic band syndrome, Aneuploidy are other differential diagnosis.

CONCLUSION: OEIS is a rare polymalformative syndrome possible to detect by an antenatal ultrasound. Prognosis depends on extent ion of bladder exstrophy and neural tube defects. So early prenatal diagnosis is required to give parents the option to terminate pregnancy and also helpful to plan appropriate perinatal management in less severe cases.

\section{REFERENCES:}

1. Ebert AK, Heiko Reutter, Michael Ludwig and Wolfgang H Rösch. The Exstrophy-epispadias complex. Orphanet Journal of Rare Diseases 2009; 4:23. doi:10.1186/1750-1172-4-23.

2. Smith N.M., Chambers H.M., Furness M.E., Haan E.A.- the OEIS complex: reccurence in sibs. J med Genet $1992 ; 29: 730-2$.

3. Shanske A.L., Pande S., Aref K., Vega-Ric C., Brion L., Reznik S., Timor-Tritsch I.E.- Omphaloceleexstrophy-imperforate anus-spinal defects (OEIS) in triplet pregnancy after IVF and CVS. Birth defects research (part A) $2003 ; 67: 467-71$.

4. Kallen, K., Castilla, E. E., Robert, E., Mastroiacovo, P., Kallen, B. OEIS complex--a population study. Am. J. Med. Genet 2000; 92: 62-8.

5. Austin P.F., Homsy Y.L., Gearhart J.P., Porter K., Guidi C., madsen K., maizels M.- The prenatal diagnosis of cloacal exstrophy. J Urol $1998 ; 160 ; 1179-81$.

6. Lee D.H., Cottrell J.R., Sanders R.C., Meyers C.M., Wulfsberg E.A., Sun C.C.J- OEIS complex (Omphalocele-Exstrophy-Imperforate anus-Spinal defects) in Monozygotic twins. Am J med genetics $1999 ; 84: 29-33$.

7. Keppler-Noreuil K.M.- OEIS (Omphalocele-Exstrophy-Imperforate anus-Spinal defects) : a review of 14 cases. Am J Med Genetics 2001 ; 99 : 271-9.

8. Witters I., Deprest J., Van Hole C., Hanssens M., Devlieger H., Fryns J.P.- Anogenital malformation with ambiguous genitalia as part of the OEIS complex. Ultrasound Obstet Gynecol $2004 ; 24$ : 797-8.

9. Girz B.A., Sherer D.M., Atkin J., Venanzi M., Ahlborn L., Cestone L.- First-trimester prenatal sonographic findings associated with OEIS complex ; a case and review of the literature. Am J Perinat $1998 ; 15: 15-7$.

10. Haldar A., Sharma A.K., Phadke S.R., Jain A., Agarwal S.S.- OEIS Complex with cranio-facial anomalies- defect or blastogenesis. Am J Med Genetics 1994 ; 53 : 21-3.

11. Bohring A.- OEIS complex, VATER and the ongoing difficulties in eterminology and delineation. Zm J Med genetics 2002 ; 107 : 72-6. 


\section{AUTHORS:}

1. Bharadwaj Mishra

2. Lakshmi Sujani C.H.

\section{PARTICULARS OF CONTRIBUTORS:}

1. Assistant Professor, Department of Obstetrics and Gynaecology, Asram Medical College, Eluru.

2. Post Graduate Student, Department of Obstetrics and Gynaecology, Asram Medical College, Eluru.

\section{NAME ADRRESS EMAIL ID OF THE}

\section{CORRESPONDING AUTHOR:}

Dr. Bharadwaj Mishra,

Assistant Professor,

Department of Obstetrics and Gynaecology, Asram Medical College, Eluru, A.P.

Email - bharadwaj.mishra@rediffmail.com

Date of Submission: 06/11/2013.

Date of Peer Review: 07/11/2013.

Date of Acceptance: 22/11/2013.

Date of Publishing: 28/11/2013 